\title{
Biliary stenting in advanced malignancy: an analysis of predictive factors for survival
}

\author{
This article was published in the following Dove Press journal: \\ Cancer Management and Research \\ 10 December 2014 \\ Number of times this article has been viewed
}

\author{
Mehran Afshar' \\ Koudeza Khanom ${ }^{2}$ \\ Yuk Ting $\mathrm{Ma}^{1,3}$ \\ Pankaj Punia' \\ 'Cancer Centre, Queen Elizabeth \\ Hospital Birmingham, University \\ Hospitals Birmingham NHS Trust, \\ Birmingham, UK; ${ }^{2} \mathrm{St}$ James Institute \\ of Oncology, Leeds Teaching Hospitals \\ NHS Trust, Leeds, UK; ${ }^{3}$ School of \\ Cancer Sciences, University of \\ Birmingham, Birmingham, UK
}

Purpose: Stenting of the biliary tree is a common palliative procedure to relieve obstructive jaundice in advanced malignancy. Although effective in relief of biliary obstruction and palliation of symptoms, little information is available on predictive factors for survival post-procedure. This retrospective study sought to assess factors influencing post-procedure survival in cancer patients after biliary stenting.

Methods: Case notes of all patients from a regional academic cancer center, who underwent biliary stenting for obstructive jaundice related to malignancy during 2008 and 2009 were reviewed. We collected epidemiological, biochemical, treatment and survival data on all patients. We used Kaplan-Meyer analysis to assess survival from day of first biliary stenting (adjusted for cancer types), and the Cox proportional hazards model for univariate and multivariate analysis.

Results: One hundred and ninety-four patients were included in the final analysis. Most cases were related to pancreatic cancer or cholangiocarcinoma ( 89 and 46 cases respectively). Median survival for all patients was 143 days. In multivariate analysis serum albumin $\geq 34 \mathrm{~g} / \mathrm{L}$ at the time of procedure (hazard ratio $0.573 ; 95 \%$ confidence interval $0.424-0.773, P<0.001$ ) and chemotherapy post-stent (hazard ratio 0.636 ; $95 \%$ confidence interval $0.455-0.889, P=0.008$ ) were two independent prognostic factors predicting a better survival post-stenting. The 30 day mortality post-procedure in the 194 patients was $12 \%$.

Conclusion: This study suggests that stenting of the biliary tree in cases of malignant obstruction allows durable palliation of symptoms even in cases where further active chemotherapy treatment is not possible. However, the better outcome observed in patients with albumin $\geq 34 \mathrm{~g} / \mathrm{L}$ and those receiving chemotherapy post-stent requires further validation.

Keywords: jaundice, cancer, biliary stent, survival

\section{Introduction}

Advanced stage malignancy can cause obstructive jaundice related to primary cancers originating near the porta-hepatis (cholangiocarcinoma, pancreatic cancer) or cancers commonly metastasizing to the liver (colorectal cancers, gastric cancer). Untreated, this can lead to a significant loss in quality of life and complications such as bacterial cholangitis, liver failure or pain, which may all contribute to early death.

A potentially curative option in this situation is surgery, ${ }^{1,2}$ which is possible in a small number of patients. However, in the majority of cases surgery or biliary drainage is instigated as a palliative procedure for symptom control and to improve quality of life. ${ }^{3,4}$ A limited number of patients may benefit from chemotherapy but it is not possible to give chemotherapy with obstructive jaundice due to the hepatic metabolism of many chemotherapeutic agents and the poor performance status of a significant
Correspondence: Pankaj Punia

Cancer Centre, Queen Elizabeth Hospital Birmingham, University Hospitals Birmingham NHS Trust, Edgbaston, Birmingham BI5 2TH, UK

Tel +44 I2I 37| 357| ext I357|

Fax +44 I2I 37I 3590

Email pankaj.punia@uhb.nhs.uk 
proportion of patients. ${ }^{5}$ In such patients endoscopic or percutaneous biliary stent placement is a standard palliative procedure to relieve obstruction, palliate symptoms, and to improve quality of life. . $^{6-9}$

Although a body of evidence exists in the literature confirming the effectiveness of this procedure for relief of biliary obstruction and subsequent palliation of symptoms in some patients, ${ }^{2,3}$ little information is available on the long-term benefit to patients and predictive factors for survival outcomes after relieving obstructive jaundice. For the oncologist, who is the member of the multidisciplinary team usually responsible for referring the patient for a biliary stent, an important question is whether the patient will live long enough to truly benefit from what is an invasive procedure, and to possibly go on to receive chemotherapy. The majority of the published data focus on the technical aspects of stenting malignant biliary obstruction including the choices between types of stent. There is however a paucity of data that comprehensively addresses the key questions faced by the multi-disciplinary team in deciding whether it would be in the patient's best interests to undergo biliary stenting. Patients with advanced cancer may not benefit in terms of survival from stenting, and so the clinician must determine whether it is worth subjecting their patient to an invasive procedure during the end stages of their life. This decision is based on numerous factors, but would be significantly aided by an ability to pre-determine the likelihood of a survival advantage for a particular patient based on measurement of validated predictive variables. Such information would also assist in the counseling of patients in order to obtain informed consent.

The main aim of this study is to assess the survival outcomes for patients with malignant biliary obstruction after biliary stenting and to look for predictive factors that may guide better patient selection.

\section{Materials and methods}

Paper case notes and electronic patient records of all patients who underwent biliary stenting for obstructive jaundice related to malignant disease from January 1, 2008 to December 31, 2009 at St James Institute of Oncology in Leeds UK, were retrospectively analyzed. In this large tertiary center 534 biliary stents were inserted during the time period, of which 289 were for malignant disease, accounting for a total of 220 patient notes reviewed in this study. Patients without advanced malignancy, and those who underwent curative surgery were excluded from our analysis. Data were collected in relation to first stent.

Variables collected and analyzed included age, primary cancer, cause for obstruction, type of stent, number of stents, liver function tests, treatment post-stenting and survival data from the date of stenting. Patients were divided into groups for age (below and above 75), chemotherapy post-stenting, and albumin level $(>34 \mathrm{~g} / \mathrm{L}$ is considered normal as per our local reference ranges). Performance status data at the time of stenting were not available for the majority of patients. There were 184 deaths in the cohort, which meant there was adequate power to reliably investigate factors for their correlation with overall survival.

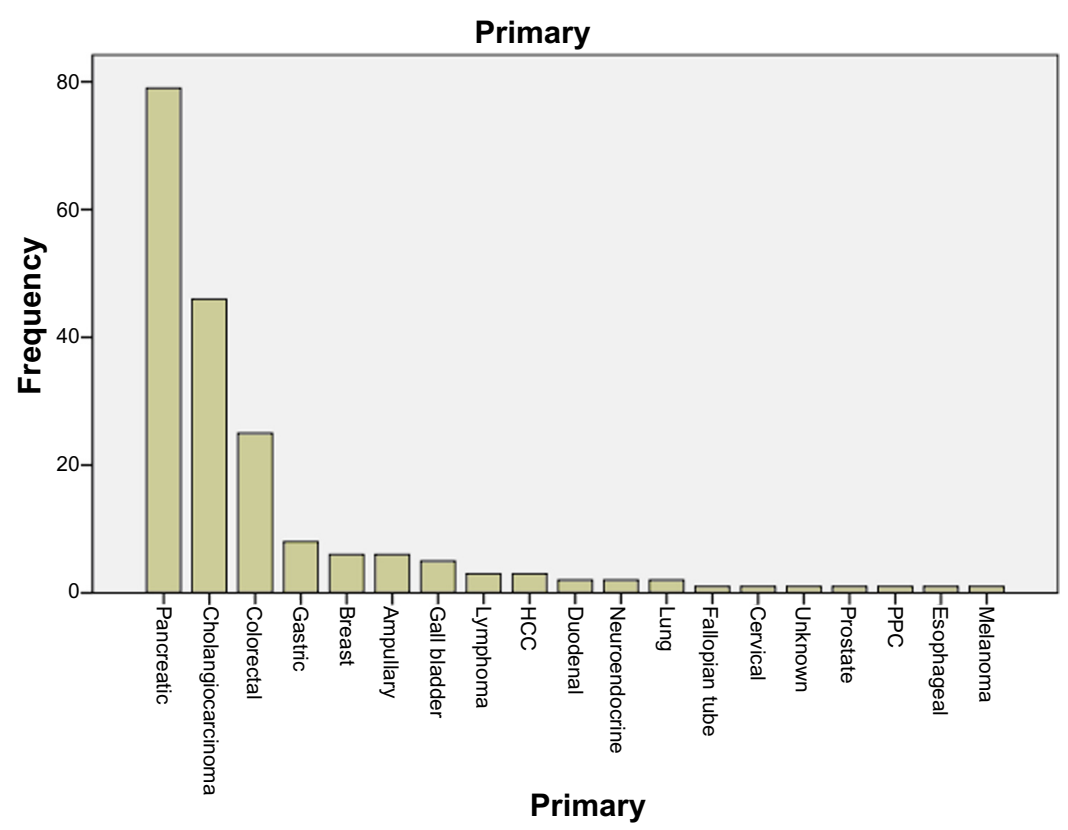

Figure I Frequency of cancer types treated.

Abbreviations: PPC, primary peritoneal cancer; HCC, hepatocellular carcinoma. 
Kaplan-Meyer analysis was used to calculate median survival from the day of first procedure (adjusted for cancer type). After initial univariate analysis, a multivariate analysis of factors that were found to have a statistically significant impact on outcomes was carried out using the Cox regression model. A $P$-value of $<0.05$ was considered significant.

\section{Results}

Out of 220 patients, 194 patients with advanced malignancy were included in our final analysis for predictive factors due to the palliative nature of their treatment. Twenty-six patients who had curative surgery for their cancer were excluded from our final analysis.

The median age of patients undergoing palliative biliary stenting was 69 years (range 24-95) (Table 1). In the cohort

Table I Patient characteristics of final cohort analyzed (194 patients)

\begin{tabular}{ll}
\hline Groups & Number (\%) \\
\hline Age & \\
$<75$ & $134(70 \%)$ \\
$>75$ & $60(30 \%)$ \\
Albumin & \\
$\quad$ Low & $93(48 \%)$ \\
Normal & $98(51 \%)$ \\
Not available & $3(1.5 \%)$ \\
Stent type & \\
Plastic & $108(56 \%)$ \\
Metal & $86(44 \%)$ \\
Cause of obstruction & \\
Primary & $144(74 \%)$ \\
Metastatic & $50(26 \%)$ \\
Treatment after stent & \\
Chemotherapy & $59(30 \%)$ \\
No chemotherapy & $135(70 \%)$ \\
\hline
\end{tabular}

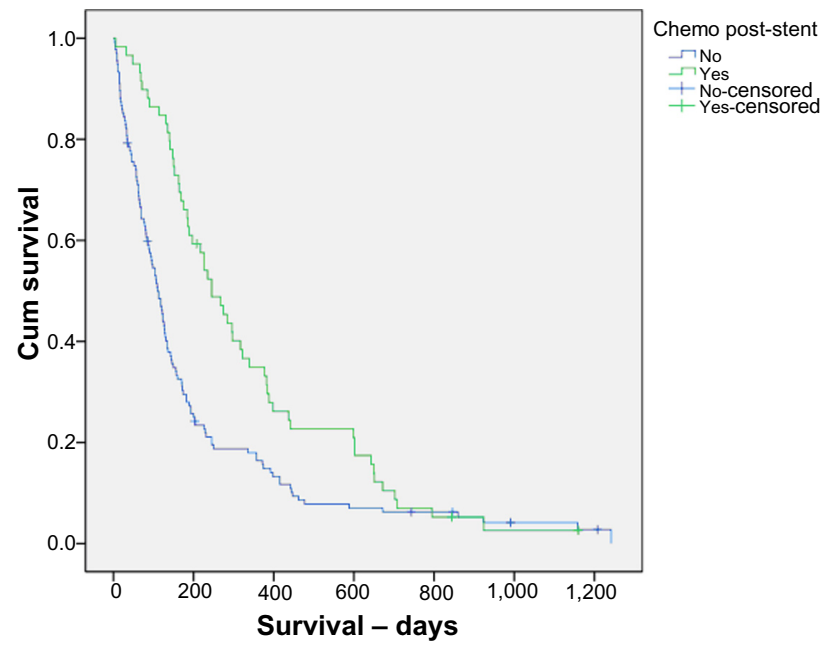

Figure 3 Kaplan-Meier curve showing survival in days for patients who received chemotherapy post-stent and those who did not.

Abbreviation: Cum, cumulative.

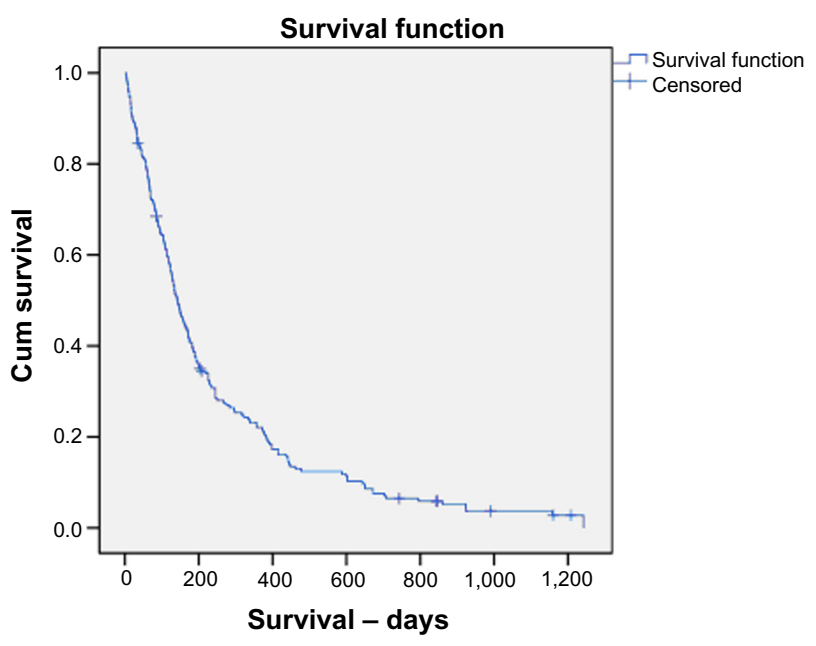

Figure 2 Kaplan-Meier curve showing survival in days of all patients within the cohort in the final analysis.

Note: The median survival was 143 days (95\% confidence interval, II2-175).

Abbreviation: Cum, cumulative.

there were 19 different primary malignancies, but the majority of cases were either pancreatic $(n=79,41 \%)$ or cholangiocarcinoma $(n=46,24 \%)$. One hundred and eight $(56 \%)$ patients had a plastic stent in comparison to $86(44 \%)$ patients who had metal stents (Table 1 and Figure 1). The majority of metal stents were placed at the time of the second procedure due to failure or occlusion of the plastic stent. Twenty (10.3\%) patients had percutaneous transhepatic cholangiography (PTC), mainly due to technical reasons. Mean time between repeat procedures after plastic stent was 113 days.

\section{Survival}

Survival analysis was carried out for the primary cancer type, cause of obstruction, type of stent, and the whole patient

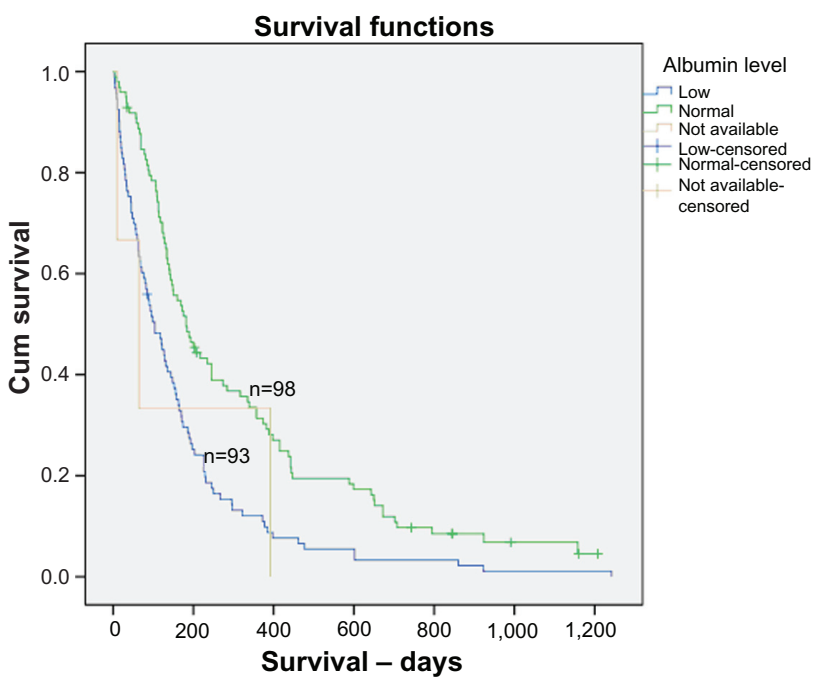

Figure 4 Kaplan-Meier curve showing survival in days for patients with low albumin levels $(<34 \mathrm{~g} / \mathrm{L})$ in comparison to patients with normal albumin levels. Abbreviation: Cum, cumulative. 
Table 2 Multivariate analysis of predictive factors for survival

\begin{tabular}{lllll}
\hline Predictive factor & $P$-value & $\begin{array}{l}\text { Hazard } \\
\text { ratio (HR) }\end{array}$ & $\begin{array}{l}\mathbf{9 5 . 0 \%} \\
\text { confidence } \\
\text { interval for HR }\end{array}$ \\
\cline { 3 - 5 } & & & Lower & Upper \\
\hline Albumin level $(\geq 34)$ & $<0.000$ & 0.573 & 0.424 & 0.773 \\
Age group $(>75)$ & 0.066 & 0.739 & 0.535 & 1.020 \\
Chemo post-stent & 0.008 & 0.636 & 0.455 & 0.889 \\
\hline
\end{tabular}

group. One hundred and eighty-four out of 194 patients had died at the time of analysis.

Median survival across all cancer types was 143 days (95\% confidence interval 112-175) (Figure 2). Patients with obstructive jaundice related to primary cancer had a median survival of 144 days in comparison to 140 days for patients with obstruction secondary to metastatic cancer. Median survival in the subgroup where obstructive jaundice was related to metastatic GI cancer (colorectal, duodenal, gastric and esophageal cancers, total number - 37) was 146 days. Median survival was also similar, regardless of stent type (plastic - 135 days versus metal - 144 days). Thirty day mortality rate post-palliative procedure was $12 \%$, mainly related to disease progression.

\section{Predictive factors}

Patients who were fit to have chemotherapy post-stenting had a much better median survival of 245 days compared to patients who did not have any chemotherapy after stenting, with median survival of 110 days $(P<0.0001)$ (Figure 3$)$. This was also the case in the GI cancer subgroup, where median survival in patients receiving chemotherapy after stenting was 235 days in comparison to 123 days in the no chemotherapy group.

\section{Gl subgroup}

In univariate analysis albumin level $(\geq 34 \mathrm{~g} / \mathrm{L})$ at time of stent ( $P \leq 0.001)$, age at the time of stent $(<75$ years $)(P=0.004)$, and chemotherapy after the stent $(P \leq 0.001)$ came out as strong predictors of better survival after biliary stenting. Bilirubin $(P=0.303)$, ALT $(P=0.562)$, ALP $(P=0.588)$, type of stent $(P=0.08)$, and cause of obstruction $(P=0.337)$ were not found to have a statistically significant effect on overall survival in univariate analysis.

Multivariate analysis showed that albumin level $\geq 34 \mathrm{~g} / \mathrm{L}$ (hazard ratio 0.557; 95\% confidence interval 0.424-0.773, $P<0.001$ ) (Figure 3 and 4 ) and chemotherapy post-stenting (hazard ratio $0.453 ; 95 \%$ confidence interval $0.455-0.889$, $P=0.008)$ were independent predictors for improved survival
(Table 2). Better survival outcomes in both the high albumin level and chemotherapy groups were statistically significant.

\section{Discussion and conclusions}

Obstructive jaundice related to primary or metastatic cancer is very common in advanced malignancy. It may be related to diffuse parenchymal infiltration of the liver or extra-hepatic biliary compression. Jaundice may have therapeutic implications as most chemotherapy drugs used in palliative cancer management have hepatic or renal metabolism. The overarching aims of biliary drainage in malignancy are palliation of symptoms, improvement in quality of life, prevention of complications like sepsis, and to improve chances of delivering palliative chemotherapy based on performance status and available treatments. ${ }^{8,9}$ There is plenty of evidence available to support this procedure for palliation of symptoms ${ }^{3}$ in different patient groups, but there is little evidence to suggest long-term outcomes after stenting, or predictive factors that may identify patients more likely to benefit for better selection. An ability by the treating clinician to positively delineate patient subgroups in order to select those deemed suitable for what is an invasive procedure will be beneficial for patients and for the sustenance of strained health care resources.

Only one other study exists in the literature that has attempted to analyze prognostic factors in this setting. That study concentrated on tumor histology as having a significant impact on survival. ${ }^{10}$ However, the small numbers of patients with each type of cancer in that study would make such an analysis less statistically robust. This retrospective study is the largest of its kind to date, and demonstrates that the most important factors in predicting long-term outcomes after biliary stenting are albumin level at the time of stenting and patient fitness to receive chemotherapy after their procedure. Patient fitness at the time of chemotherapy also implies good World Health Organization performance status as patients should normally be performance status $0-2$ to receive chemotherapy. One of the limitations of this study was that we were unable to collect accurate data about performance status from clinical documentation to use in our analysis. Age $\geq 75$ years was also a predictor of poor outcome in univariate analysis but it was not statistically significant in multivariate analysis. This may be related to the small number of patients in this sub-group. Metal stent insertion appeared to impact outcomes with regards to fewer subsequent interventions but did not affect overall survival outcomes in our study. Overall 30 day mortality post-procedure was $12 \%$; this was probably multifactorial 
with progression of cancer being the main contributing factor. Another evident limitation of this study is its retrospective nature. We propose that further validation of the predictive factors identified here is warranted.

Our study clearly suggests that relieving jaundice improves survival in patients, even if they are not fit for chemotherapy. This in itself is an important finding, as little data exist in this setting, and such a suggestion will aid the decision making process for the multidisciplinary team. Median survival in the "no chemo group" in this study after biliary stenting was 110 days. We do not have data for patients who did not undergo biliary stenting but documented survival times in the literature for these patients is in the range of 3-4 weeks. ${ }^{11}$ We have suggested certain predictive variables that may be useful in the clinical setting for stratifying patients into treatment groups, but these findings will need further validation.

\section{Disclosure}

The authors have no conflicts of interest to disclose.

\section{References}

1. Domanskii BV, Vasil'chuk AV, Umanets VS. [Surgical treatment of patients with obstructive jaundice]. Klin Khir. 1992;(2):20-23. Russian.
2. Taylor MC, McLeod RS, Langer B. Biliary stenting versus bypass surgery for the palliation of malignant distal bile duct obstruction: a meta-analysis. Liver Transpl. 2000;6(3):302-308.

3. Glazer ES, Hornbrook MC, Krouse RS. A Meta-Analysis of Randomized Trials: Immediate Stent Placement vs Surgical Bypass in the Palliative Management of Malignant Biliary Obstruction. J Pain Symptom Manage. 2014;47(2):307-314.

4. Van Laethem JL, De Broux S, Eisendrath P, Cremer M, Le Moine O, Devière J. Clinical impact of biliary drainage and jaundice resolution in patients with obstructive metastases at the hilum. Am J. Gastroenterol. 2003;98(6):1271-1277.

5. Nakao I. [Hepatic failure and renal failure in antineoplastic chemotherapy, and its treatment]. Gan To Kagaku Ryoho. 1986;13(10):2930-2938. Japanese.

6. Ferreira LE, Baron TH. Endoscopic stenting for palliation of malignant biliary obstruction. Expert Rev Med Devices. 2010;7(5):681-691.

7. Garcea G, Ong SL, Dennison AR, Berry DP, Maddern GJ. Palliation of malignant obstructive jaundice. Dig Dis Sci. 2009;54(6): 1184-1198.

8. Meller MT, Arts GR, Dean JR. Outcomes in percutaneous stenting of non-hepato-biliary/pancreatic malignant jaundice. Eur J Cancer Care (Engl). 2010;19(5):664-668.

9. Travis TSN Palliation of unresectable pancreatic malignant biliary obstruction: Results of a randomized trial comparing percutaneously placed metal and plastic endoprostheses. J Interv Radiol. 1997;12:17-21.

10. Abali H, Sezer A, Oğuzkurt L, et al. Which patients with advanced cancer and biliary obstruction benefit from biliary stenting most? An analysis of prognostic factors. Support Care Cancer. 2013;21(4):1131-1135.

11. Kasuga A, Ishii H, Ozaka M, et al. Clinical outcome of biliary drainage for obstructive jaundice caused by colorectal and gastric cancers. Jpn J Clin Oncol. 2012;42(12):1161-1167.
Cancer Management and Research

\section{Publish your work in this journal}

Cancer Management and Research is an international, peer-reviewed open access journal focusing on cancer research and the optimal use of preventative and integrated treatment interventions to achieve improved outcomes, enhanced survival and quality of life for the cancer patient The journal welcomes original research, clinical \& epidemiological

\section{Dovepress}

studies, reviews \& evaluations, guidelines, expert opinion \& commentary, case reports \& extended reports. The manuscript management system is completely online and includes a very quick and fair peerreview system, which is all easy to use. Visit http://www.dovepress.com/ testimonials.php to read real quotes from published authors. 